\title{
Association of regional body fat with metabolic risks in Chinese women
}

\author{
Xiaohua Fu', ${ }^{1,}$ Aihua Song ${ }^{1}$, Yunjie Zhou' ${ }^{1}$, Xiaoguang $\mathrm{Ma}^{3}$, Jingjing Jiao ${ }^{1,4}$, \\ Min Yang ${ }^{1,4}$ and Shankuan Zhu ${ }^{1,4, *}$ \\ 'Obesity and Body Composition Research Center, Chronic Disease Research Institute, Zhejiang University, \\ Hangzhou, People's Republic of China: ${ }^{2}$ Department of Reproductive Endocrinology, Zhejiang Provincial \\ Hospital, Hangzhou, People's Republic of China: ${ }^{3}$ Department of Epidemiology and Biostatistics, Arnold School \\ of Public Health, University of South Carolina, Columbia, SC, USA: ${ }^{4}$ Department of Nutrition and Food Hygiene, \\ Zhejiang University School of Public Health, 866 Yu-hang-tang Road, Hangzhou, Zhejiang 310058, People's \\ Republic of China
}

Submitted 16 January 2013: Final revision received 24 August 2013: Accepted 27 August 2013: First published online 22 October 2013

\begin{abstract}
Objective: To investigate the association of regional fat depots with metabolic risk factors in Chinese women.

Design: Total and regional fat depots including android fat and gynoid fat were measured by dual-energy X-ray absorptiometry. Central fat distribution was defined as android:gynoid fat ratio. Metabolic risk factors were defined as elevated TAG, reduced HDL-cholesterol, elevated blood pressure and elevated fasting plasma glucose. Logistic regression analyses were performed to examine the associations of regional fat depots with metabolic risk factors. The odds ratios of metabolic risks were further calculated according to tertiles of android fat and gynoid fat.

Setting: Participants were recruited from a community-based cross-sectional study. Face-to-face questionnaires, anthropometric and dual-energy X-ray absorptiometry measures were conducted.

Subjects: Chinese women ( $n$ 609) aged 18-79 years.

Results: Android fat and android:gynoid fat ratio were associated with significantly increased odds $(\mathrm{OR}=1 \cdot 4-3 \cdot 7 ; P<0 \cdot 01)$ for almost all risk factors, whereas gynoid fat was independently associated with significantly decreased odds $(\mathrm{OR}=0 \cdot 3-0 \cdot 6 ; P<0 \cdot 01)$. The inverse associations of gynoid fat with metabolic risk factors remained after adjusting for android fat. Even if their android fat level was in high, women in the highest tertile of gynoid fat had lower odds of having at least two metabolic risk factors compared with women in the lowest gynoid fat tertile $(P$ for trend $<0 \cdot 01)$.

Conclusions: There were opposite associations of android and gynoid fat with metabolic risks in Chinese women. Gynoid fat rather than android fat might be a more important inclusion in metabolic disease risk evaluation in female Asians.
\end{abstract}

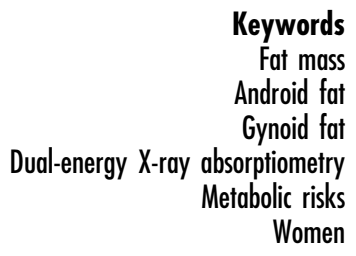

Keywords

Android fat

Gynoid fat

Metabolic risks

Women
Obesity is a complex heterogeneous disorder which is strongly associated with multiple metabolic dysfunctions including dyslipidaemia, impaired glucose tolerance and arterial hypertension ${ }^{(1,2)}$. Although previous studies have documented the association between obesity and metabolic diseases, there is increasing evidence suggesting that the presence of excess fat in specific regional body areas is a more significant indicator than total fat volume ${ }^{(3-6)}$. The excess accumulation of central fat, even without manifestations of general obesity, is closely associated with metabolic disturbances that indicate increased risks of CVD and diabetes ${ }^{(7-9)}$ whereas peripheral subcutaneous fat may be relatively benign with respect to metabolic disease risks ${ }^{(10-12)}$. For instance, the Quebec Family Study showed that larger hip circumference was inversely associated with several cardiovascular risk factors, including high TAG and insulin concentrations ${ }^{(13)}$. Therefore, collection of information on fat regional distribution patterns may add more insights in understanding the considerable metabolic variation among individuals.

Women are more likely to store fat in the lower body (around the hips and bottom, or gynoid region) and are more susceptible to age-related fat distribution change than men $^{(14)}$. The CVD risk is much lower in premenopausal women than in age-matched men, although their overall adiposity is much greater than men's ${ }^{(15)}$. However, with the 
remarkable fat redistribution that occurs after middle age, or especially after the menopause, the risk of CVD increases sharply in women ${ }^{(16,17)}$. This body composition change includes an increase in total-body and central-area (android region) fat, and a decrease in the proportion of gynoid fat. However, little is known about how and to what extent regional fat and its distribution pattern are associated with CVD progression for women. Furthermore, disparities in such associations might exist among ethnic groups ${ }^{(18)}$. Compared with their Western counterparts, Asian women have a greater increment of upper-body fat store with age, which is $\sim 0.33 \mathrm{~kg} / 10$ years in Asian women $v . \sim 0.07 \mathrm{~kg} /$ 10 years in Caucasian or African-American women ${ }^{(19)}$. However, evidence regarding the association of regional fat depots with the outcomes of metabolic disorders in this subgroup is limited ${ }^{(20)}$.

To elucidate these issues, the present study aimed to explore the association of regional body fat, indicated as android fat and gynoid fat from dual-energy X-ray absorptiometry (DXA), with metabolic risk factors in a community-based study of Chinese women. We are particularly interested to know if accurate measurement of site-specific body fat may provide a more thorough understanding of metabolic disease characteristics in Chinese women.

\section{Materials and methods}

\section{Participants}

A total of 627 women aged from 18 to 79 years old were voluntarily recruited through leaflets and posters between November 2008 and May 2009. Measurements were conducted at the Obesity and Body Composition Research Center of Zhejiang University School of Public Health. The study was designed to establish a community-based database on obesity-related chronic disease. Individuals with known malignancies, thyroid disease, pituitary disorders or hypogonadism were not eligible. Fourteen women with missing DXA information and another four women with missing data from the blood test were also excluded. In the end, 609 women were included in the analysis. In addition, none of the women had ever received hormone replacement therapy. The study was conducted according to the guidelines laid down in the Declaration of Helsinki and all procedures involving human subjects were approved by the Ethics Committee of the Second Affiliated Hospital of Zhejiang University. Written informed consent was obtained from each participant.

\section{Variable definition}

Comprehensive questionnaires on lifestyle, medical history and menopause were conducted face-to-face at the research centre. In terms of smoking status, participants were classified into two categories: (i) smokers, who are either current smokers or past smokers; and (ii) non-smokers, who never smoked. Drinking level was categorized as heavy, moderate and never. Heavy drinkers were defined as those who drank beer, wine or hard liquor no less than one time per day during the past month. Moderate drinkers were those who consumed an alcoholic beverage less than once per day during the past month. Occupational physical activity was classified into four categories: (i) 'low activity' such as office work; (ii) 'moderate activity' such as driver; (iii) 'high activity' such as farming or steelmaking; and (iv) 'other', which refers to participants with missing information on occupational activity ${ }^{(21,22)}$. Leisuretime physical activity (LTPA) was categorized as none, $0<\mathrm{LTPA}<150 \mathrm{~min} /$ week, or $\mathrm{LTPA} \geq 150 \mathrm{~min} /$ week $^{(23)}$. Menopause was defined if there had been complete cessation of menses for more than 12 months.

\section{Anthropometric measures and blood pressure}

Anthropometric measurements were obtained based on a standardized protocol. To prevent any hypoglycaemia accidents, all anthropometric measurements were conducted after a simple breakfast including a piece of bread and a bottle of yoghurt. Height was measured without shoes to the nearest $0 \cdot 1 \mathrm{~cm}$, while weight with only light clothing was measured to the nearest $0 \cdot 1 \mathrm{~kg}$ (Detecto, Webb City, MO, USA). BMI was calculated as body weight (in kilograms) divided by the square of height (in metres). Blood pressure (BP) was measured using a standard mercury sphygmomanometer after the participant had rested for at least $10 \mathrm{~min}$. All values were recorded as the mean of three measurements.

\section{Laboratory measurements}

All blood samples were obtained from the participants after a requested $12 \mathrm{~h}$ fast. Fasting plasma glucose (FPG) concentrations were determined using the hexokinase method, TAG concentrations using the enzymatic method and HDL-cholesterol (HDL-C) concentrations were determined using an enzymatic homogeneous assay. All reagents were obtained from Roche (Roche Diagnostics, Indianapolis, IN, USA).

\section{Fat measurements}

DXA (software version 11·40·004; GE Lunar Prodigy, Madison, WI, USA) was used to measure fat mass (FM), percentage body fat $(\% \mathrm{BF})$, android fat and gynoid fat through whole-body scans. \%BF was calculated as the ratio of FM $(\mathrm{kg})$ to body weight $(\mathrm{kg})$ multiplied by 100 . For the android region, the lower boundary was the top of the pelvis as the line of demarcation. The upper boundary was placed above the pelvis line of demarcation at a position that was equivalent to $20 \%$ of the distance between the pelvis and femoral neck. Lateral boundaries were the lines for the arms when in normal position for a whole-body scan. The gynoid region was defined with the upper boundary positioned below the pelvis cut line by 1.5 times the height of the android region. The lower boundary was positioned 


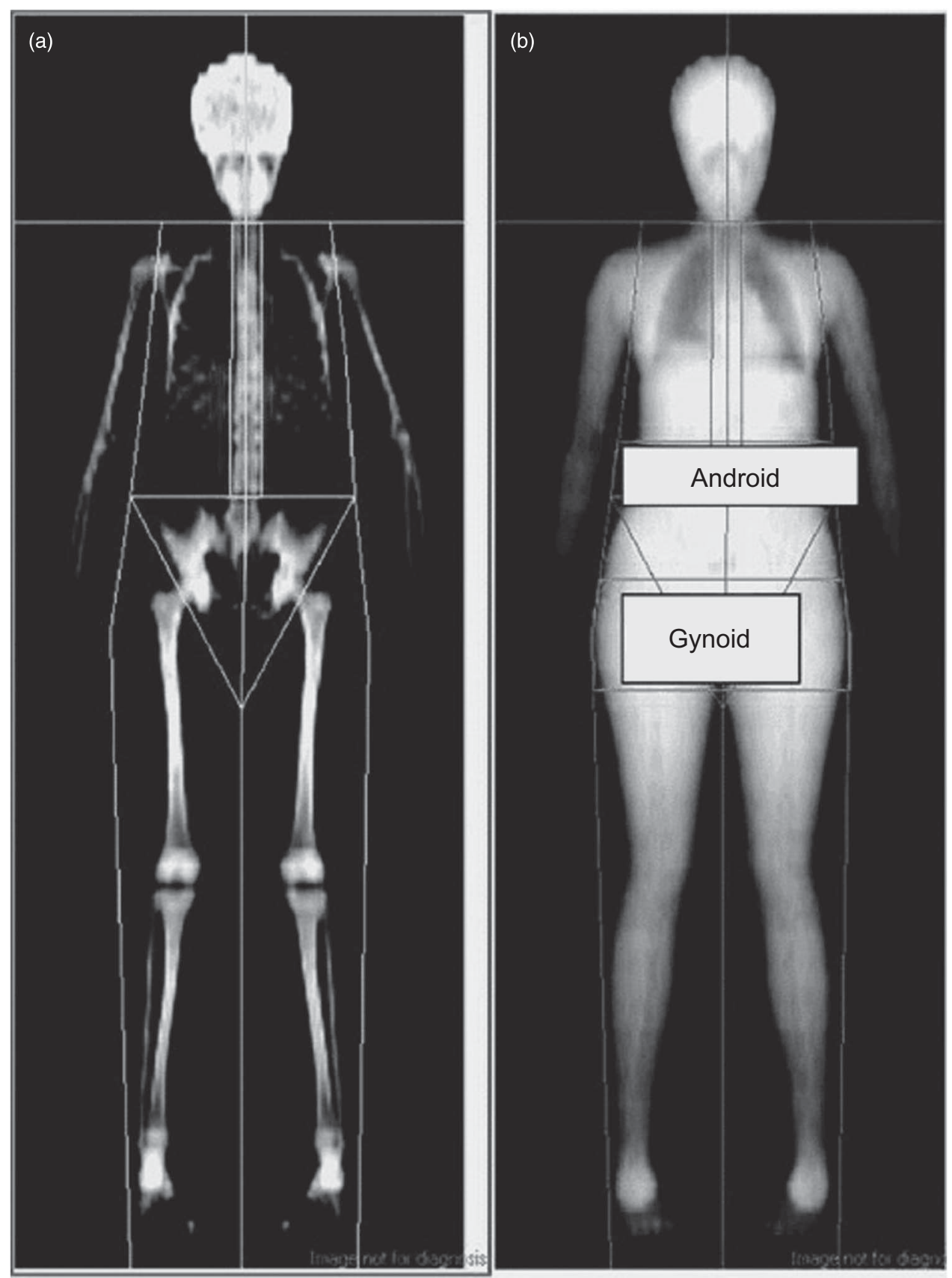

Fig. 1 Scans and regions by dual-energy X-ray absorptiometry for bone (a) and body composition (b); android, top section; gynoid, bottom section

such that it was equal to two times the height of the android region. The lateral boundaries were the outer leg lines of demarcation. Central fat distribution pattern was assessed by the android:gynoid fat ratio, as shown in Figure $1^{(24)}$. DXA was calibrated daily using a standard phantom provided by the manufacturer. Measurements were maintained within the manufacturer's precision standards of $\leq 0 \cdot 8 \%$.

\section{Metabolic risk factors}

Metabolic risk factors were defined as outcome variables including elevated TAG, reduced HDL-C, elevated BP and elevated FPG according to the recently harmonized criteria to diagnose metabolic syndrome ${ }^{(25)}$ : (i) raised TAG: $\geq 150 \mathrm{mg} / \mathrm{dl}(1.7 \mathrm{mmol} / \mathrm{l})$; (ii) reduced HDL-C: $<40 \mathrm{mg} / \mathrm{dl}$ $(1.03 \mathrm{mmol} / \mathrm{l})$ in men and $<50 \mathrm{mg} / \mathrm{dl}(1.29 \mathrm{mmol} / \mathrm{l})$ in women; (iii) raised $\mathrm{BP}$ : systolic $\mathrm{BP} \geq 130 \mathrm{mmHg}$ or diastolic $\mathrm{BP} \geq 85 \mathrm{mmHg}$; and (iv) raised FPG: $\geq 100 \mathrm{mg} / \mathrm{dl}$ $(5 \cdot 6 \mathrm{mmol} / \mathrm{l})$.

\section{Statistical analysis}

Variables were presented as means and standard deviations for continuous variables and as numbers and percentages 
for categorical variables. BMI-adjusted partial Pearson correlation analyses were performed to examine the associations of fat measures with continuous values of metabolic risk factors. Multivariate logistic regression models were used to estimate the odds ratio for each metabolic risk factor according to a $1 \mathrm{sD}$ change in fat measurement. To ensure comparability, fat measurements were expressed as a $Z$-score in the analysis. A $Z$-score is calculated as an observed value minus the sample's mean value, divided by the sample's standard deviation. In the first logistic regression analysis (model 1), the associations of each fat depot with metabolic risk factors were explored after adjusting for age, menopause, smoking, drinking, occupational physical activity and LTPA. Model 2 and model 3

Table 1 Characteristics of the study participants: Chinese women (n 609) aged 18-79 years, November 2008-May 2009

\begin{tabular}{|c|c|c|}
\hline & Mean or $n$ & SD or $\%$ \\
\hline Age (years) & $49 \cdot 8$ & $13 \cdot 3$ \\
\hline Height $(\mathrm{cm})$ & $156 \cdot 5$ & $5 \cdot 6$ \\
\hline Weight (kg) & $57 \cdot 4$ & $8 \cdot 4$ \\
\hline BMI $\left(\mathrm{kg} / \mathrm{m}^{2}\right)$ & $23 \cdot 4$ & $3 \cdot 2$ \\
\hline Menopause, $n(\%)$ & 298 & $48 \cdot 9$ \\
\hline Smokers, $n(\%)$ & 20 & $3 \cdot 3$ \\
\hline Heavy drinkers, $n(\%)$ & 47 & $7 \cdot 8$ \\
\hline Heavy activity, $n(\%)$ & 29 & $4 \cdot 8$ \\
\hline LTPA, $n(\%)$ & 165 & $32 \cdot 0$ \\
\hline \multicolumn{3}{|l|}{ Fat measures by DXA } \\
\hline FM (kg) & $18 \cdot 5$ & $5 \cdot 6$ \\
\hline$\% B F$ & $31 \cdot 6$ & $5 \cdot 9$ \\
\hline Android fat $(\mathrm{kg})$ & $1 \cdot 8$ & $0 \cdot 7$ \\
\hline Gynoid fat $(\mathrm{kg})$ & $3 \cdot 2$ & $0 \cdot 8$ \\
\hline Android:gynoid fat ratio & $0 \cdot 6$ & $0 \cdot 2$ \\
\hline \multicolumn{3}{|l|}{ Metabolic risk factors } \\
\hline TAG (mmol/l) & $1 \cdot 4$ & $0 \cdot 9$ \\
\hline HDL-C (mmol/l) & $1 \cdot 4$ & 0.3 \\
\hline Systolic BP (mmHg) & $123 \cdot 6$ & $19 \cdot 0$ \\
\hline Diastolic BP (mmHg) & $76 \cdot 4$ & $9 \cdot 9$ \\
\hline $\mathrm{FPG}(\mathrm{mmol} / \mathrm{l})$ & $5 \cdot 5$ & $0 \cdot 9$ \\
\hline
\end{tabular}

Values are presented as means and standard deviations or as numbers and percentages.

LTPA, leisure-time physical activity ( $\geq 150 \mathrm{~min} /$ week); DXA, dual-energy $\mathrm{X}$-ray absorptiometry; FM, fat mass; \%BF, percentage body fat (FM in $\mathrm{kg} /$ body weight in $\mathrm{kg} \times 100$ ); HDL-C, HDL-cholesterol; BP, blood pressure; FPG, fasting plasma glucose. were subsequently adjusted for BMI, and BMI and \%BF, respectively, on the base of model 1 . Gynoid and android fat were further mutually adjusted for each other in model 4 to determine the independent effect of body composition parameters on metabolic risk factors. Because menopause was an important confounding variable, logistic regression analyses were repeated stratified by menopause status. In addition, the tertiles of android fat and gynoid fat were calculated to estimate their associations with the risk for having at least two metabolic risk factors. The variance inflation factor and eigenvalue were used to examine the collinearity of the variables in the models. The statistical software package SPSS version $16 \cdot 0$ for Windows was used for all analyses. All statistical tests were two-tailed, and $P<0.05$ was considered significant.

\section{Results}

The characteristics and body composition of the 609 women included in the analyses are shown in Table 1. According to Chinese criteria, $3 \%$ of the participants were diagnosed as underweight (BMI $\left.<18 \cdot 5 \mathrm{~kg} / \mathrm{m}^{2}\right), \quad 52 \%$ as normal $\left(\mathrm{BMI}=18 \cdot 5-23.9 \mathrm{~kg} / \mathrm{m}^{2}\right), 45 \%$ as overweight $\left(\mathrm{BMI}=24 \cdot 0-27 \cdot 9 \mathrm{~kg} / \mathrm{m}^{2}\right)$ and none as obese $(\mathrm{BMI} \geq$ $28.0 \mathrm{~kg} / \mathrm{m}^{2}$ ). The prevalence of high TAG, low HDL-C, high BP and high FPG was $25 \cdot 5 \%, 42 \cdot 7 \%, 42 \cdot 4 \%$ and $32 \cdot 7 \%$, respectively. Participants with at least two risk factors accounted for $43.8 \%$ of the study population.

The coefficients of BMI-adjusted partial Pearson correlation of fat measures with risk factors are listed in Table 2. $\% \mathrm{BF}$, android fat and android:gynoid fat ratio were all positively correlated with age, whereas gynoid fat was negatively correlated. The coefficient of android fat with gynoid fat was low $(r=0 \cdot 18 ; P<0 \cdot 01)$, but both of them had a moderate correlation with $\% \mathrm{BF}(r=0.62$ and $r=0.55$, respectively; $P<0 \cdot 01)$. Android fat and android:gynoid fat ratio were positively correlated with TAG, systolic BP, diastolic BP and FPG, and negatively correlated with HDL-C. In contrast to this, the correlations between gynoid

Table 2 BMI-adjusted partial Pearson correlation coefficients of adiposity measures from DXA with various variables in Chinese women ( $n$ 609) aged 18-79 years, November 2008-May 2009

\begin{tabular}{|c|c|c|c|c|}
\hline & $\% B F$ & Android fat & Gynoid fat & Android:fat ratio \\
\hline Age & $0 \cdot 10^{\star}$ & $0 \cdot 24^{\star \star}$ & $-0 \cdot 31^{\star \star}$ & $0 \cdot 46^{\star \star}$ \\
\hline \multicolumn{5}{|c|}{ Body composition measures } \\
\hline$\%$ BF & $1 \cdot 00$ & $0 \cdot 62^{\star \star}$ & $0 \cdot 55^{\star \star}$ & $0 \cdot 09^{\star}$ \\
\hline Android fat & $0.62^{\star \star}$ & $1 \cdot 00$ & $0 \cdot 18^{\star \star}$ & $0 \cdot 60^{\star \star}$ \\
\hline Gynoid fat & $0.55^{\star \star}$ & $0 \cdot 18^{\star \star}$ & 1.00 & $-0.62^{\star \star}$ \\
\hline \multicolumn{5}{|c|}{ Metabolic risk factors } \\
\hline TAG & 0.04 & $0 \cdot 15^{\star \star}$ & $-0 \cdot 23^{\star \star}$ & $0 \cdot 30^{\star *}$ \\
\hline HDL-C & 0.08 & $-0.08^{\star}$ & $0 \cdot 16^{\star *}$ & $-0 \cdot 18^{\star *}$ \\
\hline Systolic BP & 0.01 & $0 \cdot 13^{\star \star}$ & $-0 \cdot 30^{\star \star}$ & $0 \cdot 34^{\star \star}$ \\
\hline Diastolic BP & 0.02 & 0.05 & $-0 \cdot 14^{\star \star}$ & $0 \cdot 13^{\star *}$ \\
\hline FPG & -0.06 & 0.08 & $-0 \cdot 26^{\star \star}$ & $0 \cdot 26^{\star \star}$ \\
\hline
\end{tabular}

DXA, dual-energy X-ray absorptiometry; \%BF, percentage body fat; HDL-C, HDL-cholesterol; BP, blood pressure; FPG, fasting plasma glucose.

${ }^{\star} P<0.05$ and ${ }^{* \star} P<0.01$. 
fat and metabolic risk factors were negative. \%BF did not have any significant correlations with metabolic risk factors.

The odds ratios and $95 \%$ confidence intervals by $1 \mathrm{SD}$ increase of each regional fat measure for metabolic risk factors are shown in Table 3. After adjusting for age, BMI, menopausal status and lifestyle-related covariates (i.e. smoking, drinking, occupational physical activity and LTPA), gynoid fat was associated with decreased odds for all risk factors (all $P<0 \cdot 01$ ) and android fat was associated with increased odds of low HDL-C $(P<0 \cdot 01)$. Android:gynoid fat ratio was independently associated with increased odds for all risk factors (all $P<0 \cdot 01$ ). The results were similar even when additionally adjusted for $\%$ BF in model 3. In model 4, when android fat and gynoid fat were included in the same model, gynoid fat was still associated with decreased odds for all metabolic risk factors (all $P<0 \cdot 001$ ).

Participants were further stratified by menopause status. Android fat and android:gynoid fat ratio were significantly higher in postmenopausal women than in premenopausal women $(P<0 \cdot 01)$, whereas gynoid fat was not different between the two groups $(P>0 \cdot 05)$. After adjusting for age, $\mathrm{BMI}, \% \mathrm{BF}$ and lifestyle covariates, the significant difference in android fat between pre- and postmenopausal women was diminished $(P>0 \cdot 05)$. Android:gynoid fat ratio was still significantly higher in postmenopausal women, and gynoid fat was significantly lower in premenopausal women than in postmenopausal women $(P<0 \cdot 01)$. The logistic models were rerun stratified by menopause status and the results were similar to those in non-stratified analysis (data not shown).

Figure 2 shows the adjusted odds for having at least two metabolic risk factors according to tertile of android fat and gynoid fat in women. The risks for having at least two risk factors increased remarkably among individuals with less gynoid fat and more android fat $(P$ for trend $<0 \cdot 01$ ). Women in the lowest tertile of gynoid fat had a threefold higher risk for having at least two risk factors than those in the highest tertile $(P<0 \cdot 01)$, when their android fat amount was in the lowest tertile. Interestingly, even when their android fat level was high (moderate or highest tertile), women in the highest tertile of gynoid fat still had lower odds for having at least two risk factors compared with those in the lowest gynoid fat tertile ( $P$ for trend $<0 \cdot 01$ ). In the multicollinearity test of the models, the variance inflation factors between adiposity measures were all $<7$ and the eigenvalues were all $>0$, both indicating that multicollinearity was not a problem in the logistic regression models.

We also did the same analysis in men ( $n$ 396) in the present study. After adjusting for age and other covariates (i.e. smoking, drinking, occupational physical activity and LTPA), \%BF, android fat, gynoid fat and android:gynoid fat ratio were all associated with increased odds for high TAG, high BP and high FPG (all $P<0 \cdot 05$ ). When additionally adjusted BMI and \%BF in the models, android fat

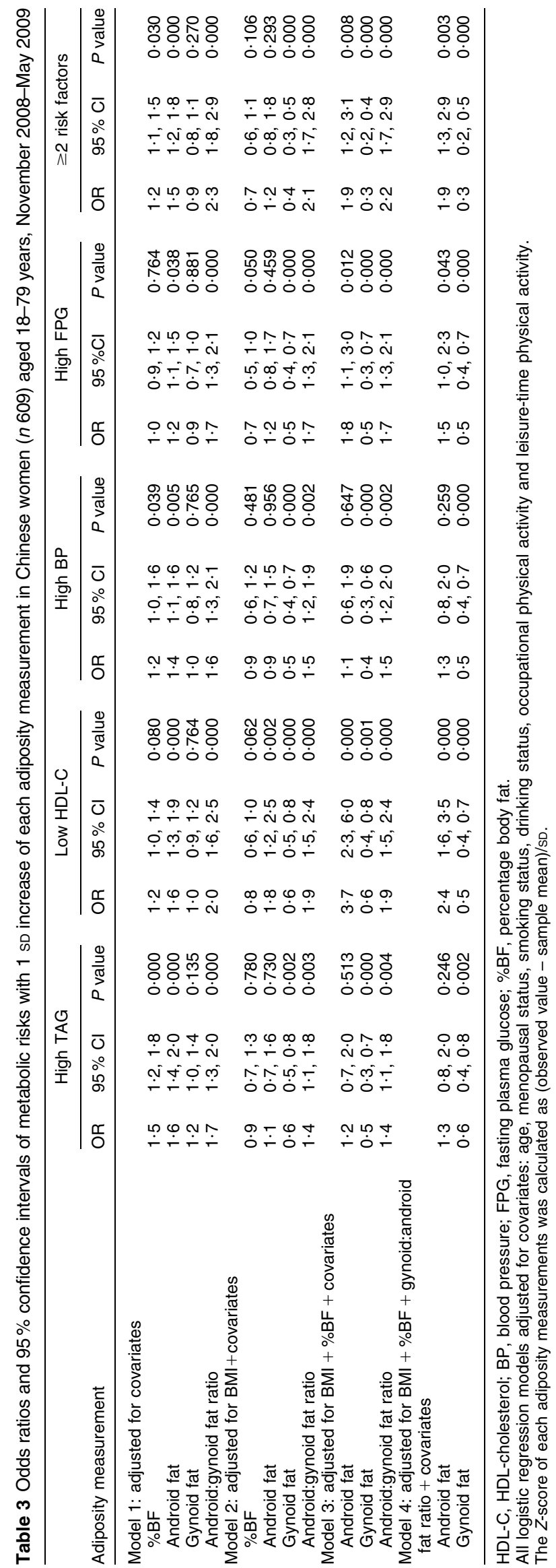




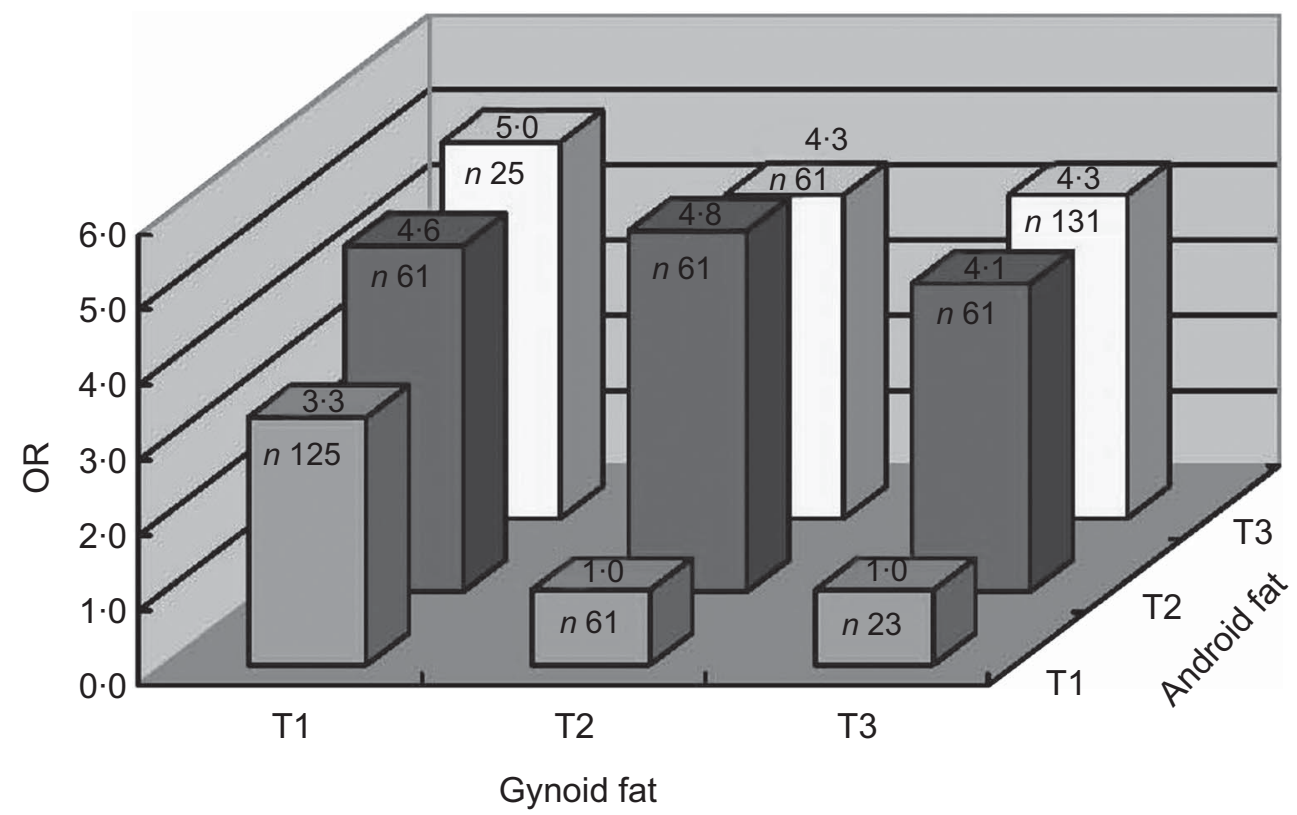

Fig. 2 Adjusted odds ratios for having at least two metabolic risk factors according to tertiles (T1, T2 and T3, where T1 is the lowest and T3 the highest) of android fat and gynoid fat in Chinese women ( $n$ 609) aged 18-79 years, November $2008-M a y 2009$. Covariates adjusted for in the analysis include age, BMI, menopausal status, smoking status, drinking status, occupational physical activity, leisure-time physical activity and percentage body fat

and gynoid fat were positively associated with high TAG, whereas android:gynoid fat ratio was positively associated with high TAG and high FPG $(P<0 \cdot 05)$. When including android fat and gynoid fat in the same model, only android fat maintained a positive association with high TAG $(P<0 \cdot 05)$; gynoid fat had no associations with any of the metabolic features (all $P>0 \cdot 05$; data not shown).

\section{Discussion}

In the current study, android fat and android:gynoid fat ratio were both significantly associated with increased odds of metabolic risk factors, whereas gynoid fat showed a decreased risk even after adjusting for android fat. In addition, compared with women in the lowest tertile of gynoid fat, those in the highest tertile had significantly lower odds of having at least two metabolic risk factors. The independently inverse association of gynoid fat with metabolic risks, in the present study, may be of great importance in Chinese women in the prevention of obesity-related diseases.

There has been much debate about which regional adiposity depot is more associated with morbidity and mortality. Previous studies demonstrated that greater waist circumference and smaller hip circumference both confer metabolic or cardiovascular risks ${ }^{(26,27)}$. However, these simple measurements cannot distinguish between fat mass and lean mass. Moreover, anthropometric measurements are subject to intra- and inter-examiner variations. Recently, more accurate methods including DXA have been of great interest. It should be noted that the measurement by DXA is of an area, rather than of one selected level as in computerized tomography, waist circumference or hip circumference, which may provide more information to understand the associations of regional fat with potential health risks ${ }^{(28-30)}$.

Previous studies conducted in Asian women demonstrated that visceral fat, rather than waist circumference, was a major determinant of metabolic risks ${ }^{(31)}$. In addition, body fat distribution, independent of general obesity, was believed to be more crucial in determining potential atherosclerotic risk factors among the healthy Chinese population $^{(32)}$. In the present study, DXA-derived android fat amount, especially android fat distribution pattern, indicated as android:gynoid fat ratio, was positively associated with metabolic abnormalities in Chinese women. Android fat region derived from DXA consists of all the fat in the upper abdomen area and around some important organs including the liver, pancreas and lower part of the heart. Compared with peripheral subcutaneous adiposity, fat accumulated in the android region is more sensitive to lipolytic stimuli, and produces more pro-inflammatory cytokines, which exerts a greater risk of developing atherothrombotic and inflammatory abnormalities associated with metabolic syndrome ${ }^{(33-35)}$. Previous studies have demonstrated that liver fat, but not visceral fat, is a better marker of the obesity-associated metabolic derangement ${ }^{(36,37)}$. Adipose tissue accumulated at the pericardial region was also found to induce endothelium dysfunction and stimulate the progression of vascular disease by releasing adipokines which acted on the 
adjacent organs and coronary vasculature ${ }^{(38-41)}$. However, studies on the association of android fat depot with metabolic diseases are very limited ${ }^{(42,43)}$. In a recent study, android fat amount was more closely associated with metabolic syndrome than abdominal visceral fat derived from computerized tomography in elderly people ${ }^{(42)}$. Another study conducted in obese children and adolescents also reported the harmful effects of android fat distribution on insulin resistance ${ }^{(43)}$. These results suggest that fat accumulation in the android area should be noticed in the pathogenesis of metabolic disturbance in addition to visceral fat.

Compared with the well-established association of central fat with disease risks, there are only a few studies regarding the association of the lower-body fat depot, which is very predominant in women, with metabolic diseases. Among the limited relevant studies, most of them reported inverse associations between hip circumference and mortality or heart diseases ${ }^{(3,27,44-51)}$. Nevertheless, hip circumference was not associated with CHD or other metabolic diseases without adjusting for waist circumference or BMI and the effect of central obesity on mortality risk could be seriously underestimated without adjusting for hip circumference ${ }^{(3,52)}$. The present study observed that the opposite associations of android fat and gynoid fat were independent of each other. In addition, according to Table 2, gynoid fat was associated with all metabolic markers whereas android fat was associated with only three of them, suggesting that gynoid fat might be a better predictor of decreased metabolic risk factors than android fat. These results agree with previous studies reporting that gluteofemoral fat, as measured by thigh circumference, hip circumference or leg adipose tissue mass, was protective and associated with lower metabolic risks, including lower LDL-cholesterol, lower TAG and higher HDL-C levels ${ }^{(53)}$. The findings of our study may be particularly crucial for Asian women, because their lower-body fat has been found to exert a greater inverse influence on insulin resistance than that in Westerners ${ }^{(18)}$.

Increased prevalence of CVD and metabolic syndrome, particularly after the menopause, has raised curiosity about the underlying factor. Previous studies suggest that the menopause transition is associated with deleterious changes in body fat distribution ${ }^{(54)}$. To be specific, an increase in android fat and a reduction in peripheral fat were observed when total fat mass had not significantly increased $^{(55)}$. These body composition changes may create a compatible environment for abnormal metabolism and aggravate cardiometabolic risk factors ${ }^{(56)}$. In our study, postmenopausal women were characterized by an absolute decreased gynoid fat and a relative increased central fat pattern. When adjusting for important variables like age, BMI and \%BF, regional fat depots were still independently associated with metabolic risk factors. This finding agrees with previous studies indicating that menopause status and the associated regional fat change are the major predictors of metabolic aberration over age in women ${ }^{(54-56)}$.

The major strength of the present study was that DXA-derived android fat and gynoid fat measures were used. Both of them were closely with traditional fat measures like waist circumference and hip circumference (the correlation coefficient between android fat and waist circumference was $0 \cdot 90, P<0 \cdot 000$; that between gynoid fat and hip circumference was $0 \cdot 82, P<0 \cdot 000$ ), whereas they are more accurate indices to understand the association of fat and fat distributions with metabolic or cardiovascular diseases. Most previous epidemiological studies have considered truncal fat as the central adiposity index ${ }^{(10,14)}$. However, the truncal fat depot can be partitioned into upper-body (android or central) and lower-body (gynoid or peripheral) areas. Mechanistically and theoretically, use of the android fat measure is more precise to manifest the impact of central fat on metabolic disease $^{(42)}$. Moreover, decreased fat in the gynoid region, which is specific for women to manifest the age-correlated fat redistribution pattern, could exert an independent influence on metabolic disorders. Lower-body obese women, despite having greater upper-body fat mass, were found to have lower NEFA release than their non-obese counterparts $^{(57)}$. These specific characteristics are important to prevent ectopic fat accumulation in women and lead to a decreased metabolic disease risk.

Limitations to the present study should be noted. First, because of the cross-sectional nature of the study, it is impossible to confirm a predictive role of regional fat on metabolic risks and subsequent incidence of diseases. Second, we failed to observe similar results in men. This may be attributable to the limited sample size in men or there might be a gender difference in the associations between fat distribution and metabolic risks. Third, BMI was a crude measure of total body size. Studies with a larger sample size are needed to analyse the associations between regional fat and metabolic risks stratified by BMI. In addition, taking the ethnic difference in the associations between regional fat measures and metabolic profile among women into account, findings from the current study may not be generalized to other ethnicities ${ }^{(58)}$.

\section{Conclusion}

Our study showed that there were opposite associations of android and gynoid fat with metabolic risks in Chinese women. Gynoid fat rather than android fat might be more important for inclusion in metabolic disease risk evaluation in female Asians. The findings from the present study suggest that not only central fat but also gynoid fat should be considered in metabolic disease risk-reducing strategies in Chinese women. 


\section{Acknowledgements}

Sources of funding: This study was supported, in part, by funding from Zhejiang University and the China Medical Board (CMB) (grant 10-014). Zhejiang University and $\mathrm{CMB}$ had no role in the design, analysis or writing of this article. Conflicts of interest: All authors have no conflicts of interest in relation to the present study. Autbors' contributions: X.F. and S.Z. had full access to all of the data in the study and take responsibility for the integrity of the data and the accuracy of the data analysis. Study concept and design: X.F. and S.Z.; acquisition of data: S.Z.; statistical analysis: X.F. and S.Z.; analysis and interpretation of data: X.F., A.S., Y.Z., X.M., J.J., M.Y. and S.Z.; drafting of the manuscript: X.F. and S.Z.; critical revision of the manuscript for important intellectual content: X.F., A.S., Y.Z., J.J., M.Y. and S.Z.; obtaining funding: S.Z.; study supervision: S.Z.

\section{References}

1. Grundy SM (2004) Obesity, metabolic syndrome, and cardiovascular disease. J Clin Endocrinol Metab 89, 2595-2600.

2. World Health Organization (2000) Obesity: Preventing and Managing the Global Epidemic. Report of a WHO Consultation. WHO Technical Report Series no. 894. Geneva: WHO.

3. Zhang C, Rexrode KM, van Dam RM et al. (2008) Abdominal obesity and the risk of all-cause, cardiovascular, and cancer mortality: sixteen years of follow-up in US women. Circulation 117, 1658-1667.

4. Snijder MB, van Dam RM, Visser M et al. (2006) What aspects of body fat are particularly hazardous and how do we measure them? Int J Epidemiol 35, 83-92.

5. Bray GA, Jablonski KA, Fujimoto WY et al. (2008) Relation of central adiposity and body mass index to the development of diabetes in the Diabetes Prevention Program. Am J Clin Nutr 87, 1212-1218.

6. Despres JP \& Lemieux I (2006) Abdominal obesity and metabolic syndrome. Nature 444, 881-887.

7. Esteghamati A, Khalilzadeh O, Anvari M et al. (2009) Association of serum leptin levels with homeostasis model assessment-estimated insulin resistance and metabolic syndrome: the key role of central obesity. Metab Syndr Relat Disord 7, 447-452.

8. Masuzaki H, Paterson J, Shinyama H et al. (2001) A transgenic model of visceral obesity and the metabolic syndrome. Science 294, 2166-2170.

9. Nguyen-Duy TB, Nichaman MZ, Church TS et al. (2003) Visceral fat and liver fat are independent predictors of metabolic risk factors in men. Am J Physiol Endocrinol Metab 284, E1065-E1071.

10. Van Pelt RE, Evans EM, Schechtman KB et al. (2002) Contributions of total and regional fat mass to risk for cardiovascular disease in older women. Am J Physiol Endocrinol Metab 282, E1023-E1028.

11. Van Pelt RE, Jankowski CM, Gozansky WS et al. (2005) Lower-body adiposity and metabolic protection in postmenopausal women. J Clin Endocrinol Metab 90, 4573-4578.

12. Snijder MB, Visser M, Dekker JM et al. (2005) Low subcutaneous thigh fat is a risk factor for unfavourable glucose and lipid levels, independently of high abdominal fat. The Health ABC Study. Diabetologia 48, 301-308.
13. Seidell JC, Perusse L, Despres JP et al. (2001) Waist and hip circumferences have independent and opposite effects on cardiovascular disease risk factors: the Quebec Family Study. Am J Clin Nutr 74, 315-321.

14. Van Pelt RE, Jankowski CM, Gozansky WS et al. (2011) Sex differences in the association of thigh fat and metabolic risk in older adults. Obesity (Silver Spring) 19, 422-428.

15. Wingard DL, Suarez L \& Barrett-Connor E (1983) The sex differential in mortality from all causes and ischemic heart disease. Am J Epidemiol 117, 165-172.

16. Ley CJ, Lees B \& Stevenson JC (1992) Sex- and menopauseassociated changes in body-fat distribution. Am J Clin Nutr 55, 950-954.

17. Pascot A, Lemieux S, Lemieux I et al. (1999) Age-related increase in visceral adipose tissue and body fat and the metabolic risk profile of premenopausal women. Diabetes Care 22, 1471-1478.

18. Boutcher SH, Dunn SL, Gail Trapp E et al. (2011) Regional adiposity distribution and insulin resistance in young Chinese and European Australian women. Scand J Clin Lab Invest 71, 653-657.

19. Wu CH, Heshka S, Wang J et al. (2007) Truncal fat in relation to total body fat: influences of age, sex, ethnicity and fatness. Int J Obes (Lond) 31, 1384-1391.

20. Deurenberg-Yap M, Chew SK \& Deurenberg P (2002) Elevated body fat percentage and cardiovascular risks at low body mass index levels among Singaporean Chinese, Malays and Indians. Obes Rev 3, 209-215.

21. Lin J, Zhang M, Song F et al. (2009) Association between C-reactive protein and pre-diabetic status in a Chinese Han clinical population. Diabetes Metab Res Rev 25, 219-223.

22. Wang L (2005) Report of China Nationwide Nutrition and Health Survey 2002 (1): Summary Report. Beijing: People's Medical Publishing House.

23. Garber CE, Blissmer B, Deschenes MR et al. (2011) American College of Sports Medicine position stand. Quantity and quality of exercise for developing and maintaining cardiorespiratory, musculoskeletal, and neuromotor fitness in apparently healthy adults: guidance for prescribing exercise. Med Sci Sports Exerc 43, 1334-1359.

24. Novotny R, Going S, Teegarden D et al. (2007) Hispanic and Asian pubertal girls have higher android/gynoid fat ratio than whites. Obesity (Silver Spring) 15, 1565-1570.

25. Alberti KG, Eckel RH, Grundy SM et al. (2009) Harmonizing the metabolic syndrome: a joint interim statement of the International Diabetes Federation Task Force on Epidemiology and Prevention; National Heart, Lung, and Blood Institute; American Heart Association; World Heart Federation; International Atherosclerosis Society; and International Association for the Study of Obesity. Circulation 120, 1640-1645.

26. Shen W, Punyanitya M, Chen J et al. (2006) Waist circumference correlates with metabolic syndrome indicators better than percentage fat. Obesity (Silver Spring) 14, $727-736$.

27. Heitmann BL \& Lissner L (2011) Hip hip hurrah! Hip size inversely related to heart disease and total mortality. Obes Rev 12, 478-481.

28. Ito H, Nakasuga K, Ohshima A et al. (2003) Detection of cardiovascular risk factors by indices of obesity obtained from anthropometry and dual-energy X-ray absorptiometry in Japanese individuals. Int J Obes Relat Metab Disord 27, 232-237.

29. Lim S, Kwon SY, Yoon JW et al. (2011) Association between body composition and pulmonary function in elderly people: the Korean Longitudinal Study on Health and Aging. Obesity (Silver Spring) 19, 631-638.

30. Wang J, Thornton JC, Kolesnik S et al. (2000) Anthropometry in body composition. An overview. Ann N Y Acad Sci 904, 317-326. 
31. Hyun YJ, Kim OY, Jang Y et al. (2008) Evaluation of metabolic syndrome risk in Korean premenopausal women - not waist circumference but visceral fat. Circ J 72, 1308-1315.

32. Wu CH, Yao WJ, Lu FH et al. (1998) Relationship between glycosylated hemoglobin, blood pressure, serum lipid profiles and body fat distribution in healthy Chinese. Atherosclerosis 137, 157-165.

33. Wajchenberg BL, Giannella-Neto $\mathrm{D}$, da Silva ME et al. (2002) Depot-specific hormonal characteristics of subcutaneous and visceral adipose tissue and their relation to the metabolic syndrome. Horm Metab Res 34, 616-621.

34. Ferreira I, Snijder MB, Twisk JW et al. (2004) Central fat mass versus peripheral fat and lean mass: opposite (adverse versus favorable) associations with arterial stiffness? The Amsterdam Growth and Health Longitudinal Study. J Clin Endocrinol Metab 89, 2632-2639.

35. Ibrahim MM (2010) Subcutaneous and visceral adipose tissue: structural and functional differences. Obes Rev 11, $11-18$.

36. Magkos F, Fabbrini E, Mohammed BS et al. (2010) Increased whole-body adiposity without a concomitant increase in liver fat is not associated with augmented metabolic dysfunction. Obesity (Silver Spring) 18, 1510-1515.

37. Fabbrini E, Magkos F, Mohammed BS et al. (2009) Intrahepatic fat, not visceral fat, is linked with metabolic complications of obesity. Proc Natl Acad Sci U S A 106, 15430-15435.

38. Mazurek T, Zhang L, Zalewski A et al. (2003) Human epicardial adipose tissue is a source of inflammatory mediators. Circulation 108, 2460-2466.

39. Eringa EC, Bakker W, Smulders YM et al. (2007) Regulation of vascular function and insulin sensitivity by adipose tissue: focus on perivascular adipose tissue. Microcirculation 14, 389-402.

40. Yudkin JS, Eringa E \& Stehouwer CD (2005) 'Vasocrine' signalling from perivascular fat: a mechanism linking insulin resistance to vascular disease. Lancet 365, 1817-1820.

41. Lim S, Son KR, Song IC et al. (2009) Fat in liver/muscle correlates more strongly with insulin sensitivity in rats than abdominal fat. Obesity (Silver Spring) 17, 188-195.

42. Kang SM, Yoon JW, Ahn HY et al. (2011) Android fat depot is more closely associated with metabolic syndrome than abdominal visceral fat in elderly people. PLoS One 6, e27694.

43. Aucouturier J, Meyer M, Thivel D et al. (2009) Effect of android to gynoid fat ratio on insulin resistance in obese youth. Arch Pediatr Adolesc Med 163, 826-831.

44. Lissner L, Bjorkelund C, Heitmann BL et al. (2001) Larger hip circumference independently predicts health and longevity in a Swedish female cohort. Obes Res 9, 644-646.
45. Heitmann BL, Frederiksen P \& Lissner L (2004) Hip circumference and cardiovascular morbidity and mortality in men and women. Obes Res 12, 482-487.

46. Bigaard J, Frederiksen K, Tjonneland A et al. (2004) Waist and hip circumferences and all-cause mortality: usefulness of the waist-to-hip ratio? Int J Obes Relat Metab Disord $\mathbf{2 8}$, 741-747.

47. Canoy D, Boekholdt SM, Wareham N et al. (2007) Body fat distribution and risk of coronary heart disease in men and women in the European Prospective Investigation Into Cancer and Nutrition in Norfolk cohort: a population-based prospective study. Circulation 116, 2933-2943.

48. Yang L, Kuper H \& Weiderpass E (2008) Anthropometric characteristics as predictors of coronary heart disease in women. J Intern Med 264, 39-49.

49. Parker ED, Pereira MA, Stevens J et al. (2009) Association of hip circumference with incident diabetes and coronary heart disease: the Atherosclerosis Risk in Communities study. Am J Epidemiol 169, 837-847.

50. Heitmann BL \& Frederiksen P (2009) Thigh circumference and risk of heart disease and premature death: prospective cohort study. BMJ 339, b3292.

51. Rexrode KM, Carey VJ, Hennekens CH et al. (1998) Abdominal adiposity and coronary heart disease in women. JAMA 280, 1843-1848.

52. Cameron AJ, Magliano DJ, Shaw JE et al. (2012) The influence of hip circumference on the relationship between abdominal obesity and mortality. Int I Epidemiol $\mathbf{4 1}$, 484-494.

53. Manolopoulos KN, Karpe F \& Frayn KN (2010) Gluteofemoral body fat as a determinant of metabolic health. Int $J$ Obes (Lond) 34, 949-959.

54. Poehlman ET, Toth MJ \& Gardner AW (1995) Changes in energy balance and body composition at menopause: a controlled longitudinal study. Ann Intern Med 123, 673-675.

55. Tremollieres FA, Pouilles JM \& Ribot CA (1996) Relative influence of age and menopause on total and regional body composition changes in postmenopausal women. Am J Obstet Gynecol 175, 1594-1600.

56. Dasgupta S, Salman M, Lokesh S et al. (2012) Menopause versus aging: the predictor of obesity and metabolic aberrations among menopausal women of Karnataka, South India. J Midlife Health 3, 24-30.

57. Martin ML \& Jensen MD (1991) Effects of body fat distribution on regional lipolysis in obesity. J Clin Invest 88, 609-613.

58. Hosain GM, Rahman M, Williams KJ et al. (2010) Racial differences in the association between body fat distribution and lipid profiles among reproductive-age women. Diabetes Metab 36, 278-285. 